\title{
Binding of bovine follicular fluid glycosaminoglycans to fibronectin, laminin and low-density lipoproteins
}

\author{
R. J. Vanderboom, D. J. Carroll, M. E. Bellin, D. K. Schneider, D. J. Miller, \\ R. R. Grummer and R. L. Ax \\ Department of Dairy Science, University of Wisconsin, 1675 Observatory Drive, Madison, \\ WI 53706, USA
}

\begin{abstract}
Summary. Interactions of bovine follicular fluid glycosaminoglycans (GAGs) with extracellular matrix (ECM) components fibronectin and laminin and with low-density lipoproteins (LDL) were examined using affinity chromatography. Glycosaminoglycans from small (diameter $<5 \mathrm{~mm}$ ) and large (diameter $11-20 \mathrm{~mm}$ ) follicles were isolated from follicular fluid. The dermatan sulphate or heparan sulphate from small or large follicles was applied to Fn-, Lm- or LDL-Sepharose columns. Portions of each fraction of the bound or unbound GAG were then subjected to gel filtration h.p.l.c. for quantification. The binding interaction between dermatan sulphate and fibronectin was significantly greater than between heparan sulphate and fibronectin $(P<0.05)$; the binding interaction between GAGs from small follicles and fibronectin was significantly greater than between GAGs from large follicles $(P<0.05)$. The binding interaction between GAGs from small follicles and laminin was significantly greater than for GAGs from large follicles $(P<0.05)$. Dermatan sulphate from small follicles bound to fibronectin $(42 \%)$, laminin $(36 \%)$ and LDL $(14 \%)$ and that from large follicles bound to fibronectin (14\%), laminin (23\%) and LDL (14\%). Heparan sulphate from small follicles bound to fibronectin $(17 \%)$, laminin $(15 \%)$ and that from large follicles bound to fibronectin (13\%), laminin (10\%) and LDL (6\%). These results suggest that dermatan sulphate, but not heparan sulphate, from follicles at different stages of development exhibit a varied ability to interact with components of the ECM. Both substances bound to LDL comparably in small amounts.
\end{abstract}

Keywords: follicle; glycosaminoglycans; fibronectin; laminin; extracellular matrix; lipoproteins; cow

\section{Introduction}

Granulosa cells are hormonally responsive, undergo extensive differentiation, and play an integral role in the development of the ovarian follicle from recruitment through luteinization. At various stages of differentiation, granulosa cells contribute to the growth of the follicle by producing and secreting components of the extracellular matrix such as proteoglycans containing the glycosaminoglycans, heparan sulphate and dermatan sulphate (Ax \& Bellin, 1988), and glycoproteins such as fibronectin (Dorrington et al., 1983). In other tissue such as skin and cartilage, glycosaminoglycans (GAGs) associate with the extracellular matrix proteins fibronectin, laminin and collagen (Murata \& Yokoyama, 1987). Further, the type of GAG and the degree of sulphation of GAG that is associated with extracellular matrix components is characteristic of the age and physiological state of the tissue in which the biomolecules are located (Murata \& Bjelle, 1978, 1980; Hascall, 1981; Fransson et al., 1982; Longas et al., 1986).

At the onset of follicular recruitment, granulosa cells commence proliferation. Before FSH stimulation in vitro, granulosa cells produce and secrete fibronectin. Granulosa cell production of 
fibronectin in vitro is inhibited by addition of FSH (Skinner \& Dorrington, 1984). In contrast, production of heparan sulphate and dermatan sulphate and their associated proteoglycan cores is stimulated by FSH (Bellin et al., 1983; Ax et al., 1985). Grimek \& Ax (1982) and Grimek et al. (1984) reported differences in proteoglycan constitution between small and large follicles of cattle. Proteoglycans from small follicles contained a greater percentage of dermatan sulphate than did large follicles. Additional chemical characterization of the low molecular weight dermatan sulphate proteoglycan indicated that preparations from small follicles contained higher proportions of serine, the amino acid by which polymer side-chains of dermatan sulphate covalently attach to the protein core.

If cell-associated fibronectin functions as a physiological binding site for follicular GAGs, then it is possible that the number of GAG-binding sites on granulosa cells would decrease as the production and secretion of fibronectin by granulosa cells is inhibited. Indeed, as follicles mature, the number of binding sites for the $\left[{ }^{3} \mathrm{H}\right]$ heparin on the granulosa cells decreases (Ax et al., 1984; Bushmeyer et al., 1985; Bellin et al., 1987a). The ability of follicular GAGs to associate with granulosa cell surfaces may change as granulosa cells differentiate and follicles mature. Dermatan sulphate from large follicles is 10 times more potent at displacing [ ${ }^{3} \mathrm{H}$ ]heparin from cell membranes than is that from small follicles (Bellin et al., 1987b). Heparan sulphate from small follicles will compete for $\left[{ }^{3} \mathrm{H}\right]$ heparin-binding sites at physiological concentrations, but that from large follicles at doses up to $5 \mathrm{mg} / \mathrm{ml}$ is without effect (Bellin et al., 1987b).

Cultured bovine granulosa cells demonstrate preference for extracellular cholesterol as a source of substrate for steroidogenesis (Savion et al., 1981). Commercially available GAGs inhibited steroidogenesis by rat granulosa cells in vitro (Campbell \& Valiquett, 1982). The GAG chondroitin4-sulphate alters basal and gonadotrophin-induced secretion of progesterone by pig granulosa cells (Ledwitz-Rigby et al., 1987). Follicular fluid GAGs, at physiological concentrations, inhibit degradation of extracellular low-density lipoproteins and progesterone production by pig granulosa cells (Bellin et al., 1987a).

The purpose of this study was to determine whether follicular fluid GAGs of cattle interacted with extracellular matrix constituents or low-density lipoproteins, and whether the degree of those binding interactions was related to the size of the follicle from which the GAGs were isolated.

\section{Materials and Methods}

Isolation of follicular fluid glycosaminoglycans. Bovine ovaries were obtained from Packerland Packing Company, Green Bay, WI, USA, on three separate occasions within $30 \mathrm{~min}$ of slaughter. Follicular fluid from small (s, $<5 \mathrm{~mm}$ ) and large $(1,11-20 \mathrm{~mm})$ follicles was aspirated with a $1.0 \mathrm{ml}$ syringe fitted with a 20 -gauge needle. Antral fluid was pooled by follicular size and centrifuged $(200 \mathrm{~g}$ for $10 \mathrm{~min})$ to remove granulosa cells. The follicular fluid was frozen promptly after centrifugation and stored at $-20^{\circ} \mathrm{C}$ until dermatan sulphate and heparan sulphate were isolated by ion exchange chromatography under dissociative conditions as previously described (Bellin \& Ax, 1987).

Isolation of low-density lipoproteins. A total of 1 litre of blood was collected from the jugular vein of 3 cows in late lactation and mixed with disodium ethylenediamine tetra-acetate (EDTA) $(0 \cdot 1 \%)$, sodium azide $(0 \cdot 05 \%)$, gentamicin sulphate $(0.005 \%)$, epsilon-amino-caproic acid $(0.13 \%)$ and phenylmethyl-sulphonylfluoride $(0.26 \%)$ to inhibit coagulation and protease activity. The plasma was adjusted to a density of $1 \cdot 21 \mathrm{~g} / \mathrm{ml}$ with potassium bromide. Samples were ultracentrifuged at $112000 \mathrm{~g}$ (Beckman $50.2 \mathrm{Ti}$ rotor) for $20 \mathrm{~h}$ at $37^{\circ} \mathrm{C}$. The total lipoproteins were concentrated to a volume of $10 \mathrm{ml}$ by ultrafiltration $(50 \mathrm{ml}$ Amicon stir cell, Model 8050, Diaflow Membrane: YM10, Amicon, Danvers, MA, USA) and subjected to gel filtration chromatography (Grummer et al., 1983). Absorbance of light by each fraction was determined at $280 \mathrm{~nm}$. Because low- and high-density lipoproteins were not completely separated, the former were further purified by heparin-Sepharose affinity chromatography (Brantmeier et al., 1988). Purity of lipoprotein fractions was determined by sodium-dodecyl-sulphate-polyacrylamide gel electrophoresis (SDSPAGE) using 5 and 15\% acrylamide gels (Brantmeier et al., 1988). Protein concentration of low-density lipoproteins was estimated by Bradford analysis with a gamma-globulin standard (Bradford, 1976).

Coupling of Fn, Lm or LDL ligand to affinity chromatography columns. Plasma fibronectin from cows (Calbiochem, La Jolla, CA, USA; $M_{\mathrm{r}} 500000$; single band SDS-PAGE), or laminin from mouse tumours (Collaborative Research, Inc., Bedford, MA, USA; 95\% pure after reduction, on 7\% SDS-PAGE) was coupled to Tresyl-activated Sepharose 4B (Pharmacia, Piscataway, NJ, USA), $0.50 \mathrm{mg}$ dry weight per column. The Sepharose 4B was washed and allowed to 
swell in $50 \mathrm{ml} 1 \mathrm{mM}-\mathrm{HCl}$ for $15 \mathrm{~min}$, centrifuged $(700 \mathrm{~g}, 5 \mathrm{~min})$, and the supernatant was discarded. The Sepharose was then washed three times with $10 \mathrm{ml}$ coupling buffer $\left(1 \mathrm{M}-\mathrm{NaHCO}_{3}, 0.5 \mathrm{M}-\mathrm{NaCl}, \mathrm{pH} 8 \cdot 3\right)$.

Fibronectin or laminin $(5 \mathrm{mg})$ was dissolved in $10 \mathrm{ml}$ coupling buffer and mixed thoroughly with $1 \mathrm{ml}$ swollen Tresyl-Sepharose for $2 \mathrm{~h}$. After binding, the ligand-conjugated Sepharose was again centrifuged ( $700 \mathrm{~g}, 5 \mathrm{~min}$ ) and the supernatant was saved for protein assay. Excess ligand was washed from beads with coupling buffer, which was saved for protein assay. Unbound active sites on the Sepharose were then blocked by mixing with blocking buffer $(0 \cdot 1 \mathrm{M}$ Tris- $\mathrm{HCl}$ with $0.2 \mathrm{M}$-glycine, $\mathrm{pH} 8$ ) for $2 \mathrm{~h}$. The ligand-conjugated Sepharose beads were obtained by centrifugation and washed with three alternating cycles of acetate buffer $\left(0.1 \mathrm{M}-\mathrm{NaC}_{2} \mathrm{H}_{3} \mathrm{O}_{2} .3 \mathrm{H}_{2} \mathrm{O}\right.$ with $\left.0.5 \mathrm{M}-\mathrm{NaCl}, \mathrm{pH} 4\right)$ and Tris-washing buffer $(0.1 \mathrm{M}$-Tris buffer with $0.5 \mathrm{M}-\mathrm{NaCl}, \mathrm{pH} 8)$. The Sepharose bound to the extracellular matrix was washed a final time and equilibrated in Tris buffer $\left(0.05 \mathrm{M}\right.$-Tris $-\mathrm{HCl}, 1 \mathrm{mM}-\mathrm{MgCl}_{2}, 1 \mathrm{M}-\mathrm{CaCl}_{2}$, plus $0.01 \%$ sodium azide, $\mathrm{pH} \mathrm{7.2)} \mathrm{and} \mathrm{poured} \mathrm{into} \mathrm{a} \mathrm{column} \mathrm{consisting} \mathrm{of} \mathrm{a} 1 \mathrm{ml}$ tuberculin syringe. Subsequent use of the term 'Tris buffer' in this paper refers to this equilibration buffer.

The low-density lipoproteins were coupled to a Tresyl-activated Sepharose 4B affinity column with a procedure similar to that described above but the conjugated Sepharose was washed and separated from buffers on a sintered glass filter rather than by centrifugation. Purified low-density lipoproteins $(400 \mathrm{mg})$ were applied to $1 \mathrm{~g}$ of swollen Sepharose. The equilibrating buffer was $0.01 \mathrm{M}-\mathrm{Tr}$ is, $0.01 \mathrm{M}-\mathrm{CaCl}_{2}, 0.02 \%$ sodium azide, $\mathrm{pH} \mathrm{8.0.} \mathrm{The} \mathrm{gel} \mathrm{was} \mathrm{poured}$ into a $15 \times 35 \mathrm{~mm}$ plastic column.

Affinity chromatography of GAGs. $\left[{ }^{3} \mathrm{H}\right]$ heparin $(2 \mu \mathrm{Ci}$ in $100 \mu \mathrm{l}$ Tris buffer, New England Nuclear, Wilmington, $\mathrm{DE}, \mathrm{USA}$; sp. act. $0.34 \mathrm{mCi} / \mathrm{mg}$ ) was applied to the affinity columns to determine the binding between a commercially available GAG and the extracellular matrix ligand. After allowing a I h equilibration time, unbound $\left[{ }^{3} \mathrm{H}\right]$ heparin was eluted from the column in $10 \mathrm{ml}$ Tris buffer collected in $101-\mathrm{ml}$ fractions at a flow rate of $0.33 \mathrm{ml} / \mathrm{min}$. Bound ${ }^{3} \mathrm{H}$ ]heparin was eluted from the column with a step-wise $2 \mathrm{M}-\mathrm{NaCl}$ gradient in $10 \mathrm{ml}$ Tris buffer collected in $101-\mathrm{ml}$ fractions. Each fraction was mixed with $20 \mathrm{ml}$ scintillation cocktail (Ecoscint: National Diagnostics, Manville, NJ, USA) shaken overnight, and monitored for radioactivity for $1 \mathrm{~min}$ on a Packard Tri-Carb liquid scintillation spectrophotometer.

Samples of dermatan sulphate and heparan sulphate from large and small follicles $(100 \mu \mathrm{g})$ were loaded onto each of the fibronectin and laminin columns, and $500 \mu \mathrm{g}$ samples were loaded onto the low-density lipoproteins column, and equilibrated for $1 \mathrm{~h}$ at $4^{\circ} \mathrm{C}$. Unbound GAG was eluted from the fibronectin and laminin columns with $10 \mathrm{ml}$ Tris buffer at a flow rate of $0.33 \mathrm{ml} / \mathrm{min}$. Bound GAG was eluted from each column with $2 \mathrm{M}-\mathrm{NaCl}$ in $10 \mathrm{ml}$ Tris buffer at the same flow rate. The columns were subsequently equilibrated with $10 \mathrm{ml}$ Tris buffer. The low-density lipoprotein column was eluted initially with $25 \mathrm{ml}$ Tris buffer, followed by $50 \mathrm{ml}$ Tris buffer containing $2 \mathrm{M}-\mathrm{NaCl}$, and then re-equilibrated with $50 \mathrm{ml}$ Tris buffer. Each of those eluates was collected as a single fraction.

Samples of dermatan sulphate and heparan sulphate that did not bind to fibronectin or laminin in previous runs were subsequently applied to the extracellular matrix columns and eluted in a similar manner to confirm that overloading of the columns did not occur. This step was omitted with the low-density lipoprotein column.

Quantitation of the GAGs by h.p.l.c. Samples of each eluted fraction $(200 \mu \mathrm{l})$ were analysed for the concentration of GAG by gel filtration high performance liquid chromatography (h.p.l.c.) using a Waters Associates I-125 Protein Pak column at a flow rate of $2.0 \mathrm{ml} / \mathrm{min}$ and a run time of $12 \mathrm{~min}$. Absorbance was monitored at $205 \mathrm{~nm}$ using a Perkin-Elmer LC-75 spectrophotometer (Grimek \& Ax, 1982). Glycosaminoglycan concentrations were determined by comparison of integrated areas of known masses $(6 \cdot 25-100 \mu \mathrm{g})$ of purified heparan sulphate and dermatan sulphate standards (Bellin \& Ax, 1987).

Statistics. Each type of GAG was tested in duplicate or triplicate, with each replication representing heparan sulphate or dermatan sulphate purified from follicles harvested during separate trips to an abattoir. The amount of GAG binding to the extracellular matrix or low-density lipoprotein was expressed as $\mu \mathrm{g}$ adsorbed per $100 \mu \mathrm{g}$ loaded. A $\log$ transformation of the data was performed to normalize variance. The data were analysed using general linear models (GLM) procedures with the Statistical Analysis System (SAS Institute Inc., Box 8000, Cary, NC 27511, USA).

\section{Results}

\section{Binding of GAGs to fibronectin or laminin}

A portion of the $\left[{ }^{3} \mathrm{H}\right]$ heparin applied to the fibronectin and laminin columns did not bind $(66 \%)$ and was eluted with Tris buffer. The remaining portion of $\left[{ }^{3} \mathrm{H}\right]$ heparin did bind $(33 \%)$ to the column and was collected in the $2 \mathrm{M}-\mathrm{NaCl}$ Tris fraction (Fig. 1).

A larger proportion of GAGs from small follicles bound to the extracellular matrix components than did GAGs from large follicles $(P<0.05)$; however, the significance of this effect was due primarily to the interaction of dermatan sulphate with the extracellular matrix components from small follicles $(P<0.05$, Fig. 2). A higher percentage of dermatan sulphate from small follicles $(P<0.05)$ bound to fibronectin than did dermatan sulphate from large follicles or heparan sulphate from large and small follicles, which each bound to fibronectin in similar proportions. A 


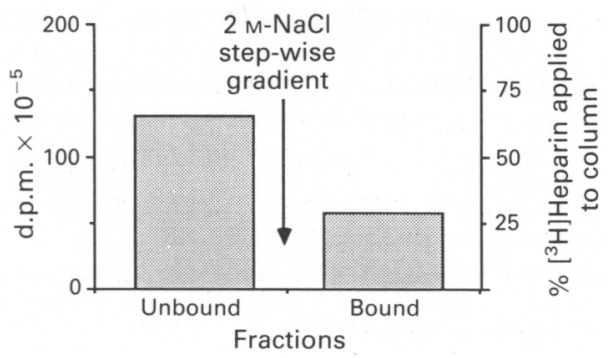

Fig. 1. The interaction of $\left[{ }^{3} \mathrm{H}\right]$ heparin with fibronectin. Unbound $\left[{ }^{3} \mathrm{H}\right]$ heparin was eluted from a fibronectin-Sepharose affinity chromatography column in 10 fractions. Radioactivity was monitored on a liquid scintillation spectrophotometer. The cumulative GAG from those fractions is depicted in the unbound column. The $\left[{ }^{3} \mathrm{H}\right]$ heparin that did bind to fibronectin was eluted from the column in a step-wise gradient of $101-\mathrm{ml}$ fractions of $2 \mathrm{M}-\mathrm{NaCl}$ in Tris buffer. The cumulative amount of GAG from those fractions is depicted in the bound column.

greater percentage of GAG from small follicles bound to laminin than did GAG from large follicles $(P<0.05$, Fig. 2). GAG which initially did not bind to fibronectin or laminin was rechromatographed and did not bind on the subsequent column run, indicating that GAG in the unbound fractions did not represent overloading of the column in the original column runs.

(a) Fibronectin

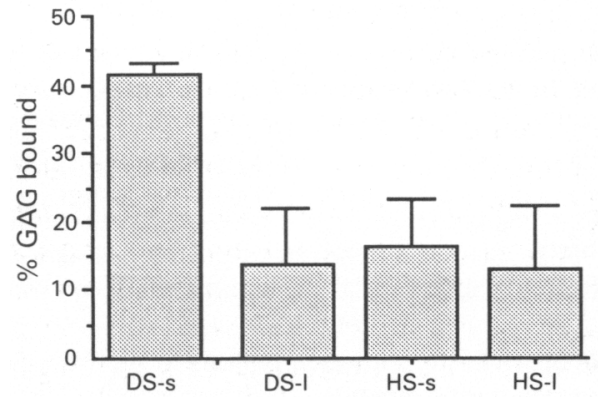

(b) Laminin

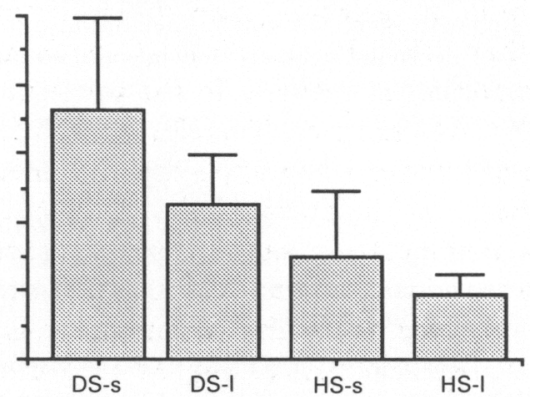

Fig. 2. The percentage of bound portions of follicular fluid glycosaminoglycans (GAG) dermatan sulphate (DS-) or heparan sulphate (HS-) from small (s) or large (l) follicles with fibronectin $(\mathrm{Fn})$ or laminin $(\mathrm{Lm})$. The values represent the mean \pm s.e. for $n=2$ GAG samples tested with Fn, and $n=3$ (DS-s and DS-l) or $n=2$ (HS-s and HS-1) for GAG samples tested with Lm. A greater amount of DS bound to the extracellular matrix proteins than did HS $(P<0.05)$. A greater amount of GAG from small follicles bound to Fn or $\mathrm{Lm}$ than did GAG from large follicles $(P<0 \cdot 05)$.

\section{Binding of GAGs to low-density lipoprotein}

Only a small proportion of each follicular fluid GAG interacted with low-density lipoprotein. For each $100 \mu \mathrm{g}$ of DS-s, DS-1 and HS-1 applied to LDL, 13.8\%, 14.2\% and $5.8 \%$ bound to the LDL, respectively. Affinity of heparan sulphate from small follicles for low-density lipoprotein was not determined due to lack of available sample. Proportions of the GAGs which bound to low-density lipoprotein were lower than binding observed with extracellular matrix constituents. 


\section{Discussion}

Glycosaminoglycans isolated from ovarian follicles interacted with components of the extracellular matrix, fibronectin and laminin and with low-density lipoprotein. Furthermore, dermatan sulphate isolated from small follicles exhibited a greater ability to bind to the extracellular matrix components than did dermatan sulphate from large follicles or heparan sulphate from either small or large follicles.

Dermatan sulphate from small follicles bound to fibronectin in higher proportions than that from large follicles. This suggests that dermatan sulphate displays different physicochemical properties at various stages of follicular maturation. That premise is supported by data from our laboratory which indicate that composite follicular GAGs isolated at different times relative to the LH surge in cattle varied in their ability to bind to fibronectin (Vanderboom et al., 1989). Since dermatan sulphate from follicles of different sizes bound fibronectin differently, it is apparent that fibronectin could potentially serve as an affinity ligand to segregate follicular fluid dermatan sulphate isomers. Fibronectin has previously been used for isolating placental proteoglycans (Isemura et al., 1987).

Dermatan sulphate and heparan sulphate account for approximately $90 \%$ and $10 \%$, respectively, of the GAGs found in follicular fluid and secreted by granulosa cells in vitro (Ax \& Bellin, 1988). The dermatan sulphate proteoglycans exist as two forms with different hydrodynamic sizes. Concentrations of the lower molecular weight forms vary with follicular maturation (Grimek \& Ax, 1982; Grimek et al., 1984), and production of a similar dermatan sulphate of low molecular weight varies when granulosa cells are exposed to gonadotrophins or cyclic nucleotides (Yanagishita \& Hascall, 1981).

The dermatan sulphate chains in the lower molecular weight proteoglycans exhibit a shift in the ratio of galactosamine to glucosamine from approximately $3: 1$ in small follicles to greater than 8:1 in large follicles (Grimek et al., 1984; Bellin \& Ax, 1987). Jensen \& Zachariae (1958) reported an increase in the proportions of galactosamine to glucosamine in composite follicular GAG samples. Therefore, the higher binding of dermatan sulphate from small than from large follicles when applied to fibronectin and laminin may be related to a subtle chemical difference in the composition of those GAG chains. Future studies should be designed to compare binding to extracellular matrix constituents of dermatan sulphate preparations with different chemical features. Additionally, dermatan sulphate chains separated from each of the higher and lower molecular weight proteoglycans as well as intact proteoglycans of both hydrodynamic sizes should be applied to fibronectin columns. Current studies are in progress to determine the chemical composition of GAGs which bind or do not bind to extracellular matrix affinity columns to determine chemical characteristics which may be important for those binding interactions.

In contrast with the variance observed in GAG-extracellular matrix interaction, little difference was noted between the interaction of GAGs isolated from follicles of different sizes and lowdensity lipoprotein in this experiment. Dermatan sulphate from immature pig follicles inhibited low-density lipoprotein degradation and progesterone production by granulosa cells in a dosedependent manner (Bellin et al., 1987a). Commercial chondroitin-4-sulphate also altered basal and gonadotrophin-induced secretion of progesterone from granulosa cells (Ledwitz-Rigby et al., 1987). Additional studies are needed to determine whether the size of follicle from which GAGs are isolated influences low-density lipoprotein degradation. Two aspects of interaction between GAGs and lipoproteins have been suggested. Glycosaminoglycans may uncouple low-density lipoproteins from cell membranes or may interact directly with them, resulting in steric hindrance or conformational changes in the apolipoprotein that prevent lipoprotein interaction with cell membrane receptors (Strauss et al., 1985). The results of the present study indicate that only a small portion of follicular GAGs interact with low-density lipoproteins with minor differences among GAGs obtained from follicles of different sizes. However, greater binding affinity was observed 
between purified follicular fluid GAGs and low-density lipoproteins than between a composite of follicular fluid GAGs and high-density lipoprotein (Brantmeier et al., 1988). In that study, approximately $2 \%$ of the GAG bound to high-density lipoprotein.

In conclusion, these studies show that follicular fluid GAG interactions with extracellular matrix proteins are dependent upon the maturity of the follicles from which GAGs are isolated. Further research should focus on specific relationships between extracellular matrix components and isomers of GAG constituents of proteoglycans that are produced in response to various hormonal stimuli. Fibronectin can serve as a potential affinity chromatography ligand to isolate specific sub-populations of GAGs, particularly dermatan sulphate, from follicular fluid for further chemical and functional characterizations.

This research was supported by the University of Wisconsin College of Agricultural and Life Sciences and USDA Grant 85-CRCR-1-1864. We thank Sue Bollig-Fenton, Tom Coyle, Kathy Horn, Shari Nass and Dale Ripley for help with the collection of ovaries and follicular fluid samples.

\section{References}

Ax, R.L. \& Bellin, M.E. (1988) Glycosaminoglycans and follicular development. J. Anim. Sci. 66 (Suppl. 2), $32-49$.

Ax, R.L., Bushmeyer, S.M., Boehm, S.K. \& Bellin, M.E. (1984) Binding of the glycosaminoglycan ${ }^{3} \mathrm{H}$-heparin to bovine granulosa cells varies with size and oestrogen content of ovarian follicles. Endocr. Res. 10, 63-72.

Ax, R.L., Bellin, M.E. \& Grimek, H.J. (1985) Properties and regulation of synthesis of glycosaminoglycans by the ovary. In Proc. Fifth Ovarian Workshop, pp. 451-480. Eds D. O. Toft \& R. J. Ryan. Ovarian Workshops, Champaign, IL.

Bellin, M.E. \& Ax, R.L. (1987) Purification of glycosaminoglycans from bovine follicular fluid. J. Dairy Sci. 70, 1913-1919.

Bellin, M.E., Lenz, R.W., Steadman, L.E. \& Ax, R.L. (1983) Proteoglycan production by bovine granulosa cells in vitro occurs in response to FSH. Molec. cell. Endocr. 29, 51-65.

Bellin, M.E., Veldhuis, J.D. \& Ax, R.L. (1987a) Follicular fluid glycosaminoglycans inhibit degradation of low density lipoproteins and progesterone production by porcine granulosa cells. Biol. Reprod. 37, $1179-1184$.

Bellin, M.E., Wentworth, B.C. \& Ax, R.L. (1987b) Comparisons of the ability of follicular fluid glycosaminoglycans and chemically desulphated heparin to compete for heparin binding sites on granulosa cells. Biol. Reprod. 37, 293-300.

Bradford, M.M. (1976) A rapid and sensitive method for quantitation of microgram quantities of protein utilizing the principle of protein-dye binding. Analyt. Biochem. 72, 248-254.

Brantmeier, S.A., Grummer, R.R. \& Ax, R.L. (1988) High density lipoproteins from bovine plasma and follicular fiuid do not possess a high affinity for glycosaminoglycans. Lipids 23, 269-274.

Bushmeyer, S.M., Bellin, M.E. \& Ax, R.L. (1985) Specific binding of ${ }^{3} \mathrm{H}$-heparin to bovine granulosa cell membranes. Molec. cell. Endocr. 42, 135-144.
Campbell, K.L. \& Valiquett, T.R. (1982) Do nonhormonal macromolecules cause correlated alterations of granulosa cell responses to FSH in vitro? Biol. Reprod. 26, (Suppl. 1), 102A, abstr.

Dorrington, J.H., McKeracher, H.L., Chan, A.K. \& Gore-Langton, R.E. (1983) Hormonal interactions in the control of granulosa cell differentiation. J. Steroid Biochem. 19, 17-32.

Fransson, L.A., Coster, L., Malmstrom, A. \& Sheehan, J.K. (1982) Self-association of sclereal proteodermal sulphate. J. biol. Chem. 257, 6333-6338.

Grimek, H.J. \& Ax, R.L. (1982) Chromatographic comparison of chondroitin-containing proteoglycan from small and large bovine ovarian follicles. Biochem. Biophys. Res. Commun. 104, 1401-1406.

Grimek, H.J., Bellin, M.E. \& Ax, R.L. (1984) Characteristics of proteoglycans from small and large bovine ovarian follicles. Biol. Reprod. 30, 397-409.

Grummer, R.R., Davis, C.L. \& Hegarty, H.M. (1983) Comparison of ultracentrifugation and gel filtration for the isolation of bovine lipoproteins. Lipids 18, 795-801.

Hascall, V.C. (1981) Proteoglycans: structure and function. In Biology of Carbohydrates, Vol. 1, pp. 1-49. Eds V. Ginsburg \& P. Robbins. Wiley, Chichester.

Isemura, M., Sato, N., Yamaguchi, Y., Aikawa, J., Munakata, H., Hayashi, N. \& Yosizawa, Z. (1987) Isolation and characterization of fibronectin-binding proteoglycan carrying both heparan sulphate and dermatan sulphate chains from human placenta. $J$. biol. Chem. 262, 8926-8933.

Jensen, C.E. \& Zachariae, F. (1958) Studies on the mechanism of ovulation. II. Isolation and analysis of acid mucopolysaccharides in bovine follicular fluid. Acta endocr., Copenh. 27, 356-364.

Ledwitz-Rigby, F., Gross, T.M., Schjeide, O.A. \& Rigby, B.W. (1987) The glycosaminoglycan chondroitin-4sulphate alters progesterone secretion by porcine granulosa cells. Biol. Reprod. 36, 320-327.

Longas, M.O., Russell, C.S. \& He, X.Y. (1986) Chemical alterations of hyaluronic acid and dermatan sulphate 
detected in aging human skin by infrared spectroscopy. Biochem. Biophys. Acta 884, 265-269.

Murata, K. \& Bjelle, A. (1978) Constitutional heterogeneity of the glycosaminoglycans in articular cartilage proteoglycans. Connect. Tiss. Res. 5, 109-116.

Murata, K. \& Bjelle, A. (1980) Constitutional variations of glycosaminoglycans in normal and arthritic bovine articular cartilage proteoglycans at different ages. Connect. Tiss. Res. 7, 143-156.

Murata, K. \& Yokoyama, Y. (1987) Dermatan sulphate isomers in human articular cartilage characterized by high-performance liquid chromatography. Biochem. Int. 15, 87-94.

Savion, N., Laherty, R., Lui, G.M. \& Gospodarowicz, D. (1981) Modulation of low density lipoprotein metabolism in bovine granulosa cells as a function of their steroidogenic activity. $J$. biol. Chem. 256, $12817-12822$.
Skinner, M.K. \& Dorrington, J.H. (1984) Control of fibronectin synthesis by rat granulosa cells in culture. Endocrinology 115, 2029-2031.

Strauss, J.F., Paavola, L.G., Nestler, J.E., Sato, E.A. \& Silavin, S.L. (1985) Lipoprotein cholesterol uptake and metabolism in ovarian cells. In Proc. Fifth Ovarian Workshop, pp. 275-302. Eds D. O. Toft \& R. J. Ryan. Ovarian Workshops, Champaign.

Vanderboom, R.J., Wise, T.H., Maurer, R.R. \& Ax, R.L. (1989) Variations in the interaction between follicular glycosaminoglycans and fibronectin relative to the LH surge in cattle. In Proc. 7th Ovarian Workshop, pp. 315-320. Ed. A. Hirshfield. Plenum Press, NY.

Yanagishita, M. \& Hascall, V.C. (1981) Biosynthesis of proteoglycans by rat granulosa cells cultured in vitro: modulation by gonadotropins, steroid hormones, prostaglandins and a cyclic nucleotide. Endocrinology 109, $1641-1649$.

Received 15 November 1988 